\title{
Grid Enabling of Nano-Science Applications in NAREGI
}

\author{
Mutsumi Aoyagi \\ Kyushu University
}

\begin{abstract}
Grid-Enabling Team(WP6) in National Research Grid Initiative(NAREGI)1 is developing application-specific middleware components to grid-enable large-scale nano-science applications, Chemistry applications, including those that require coupling of multiple applications on the grid. One example of such applications is multi-scale and/or multi-physics simulation, where each application component utilizes mathematically and physically different modeling and cooperates on spatially or temporally different calculations. To advance such multi-scale and/or multi-physics applications, users have made an every kind of efforts in developing custom codes and decomposing original codes for semantic-level communication between heterogeneous scientific application components. To facilitate easier usage and minimize customization processes of original user programs which may be legacy codes, we have been developing a middleware system, called a _Mediator_on top of the GridMPI2, that provides high-level transparency in automatically transferring and transforming data between heterogeneous application components. The Mediator focuses on a data-handling specification that correlates different discrete points in finite difference method (FDM), finite element method (FEM), or particle simulations such as Molecular Dynamics(MD) in the unified way. It supports a variety of techniques for semantically transforming the values associated with the correlated points, e.g., in-sphere, first nearest neighbors, and nearest points. The Mediator provides three types of Application Programming Interface (API), which (1) manage a task identification and construct an association between Mediator and application processes in parallel programming style such as Single Program Multiple Data and MasterWorker, (2) register different levels of discrete points, search the correlated discrete points, and determine processes and (3) transfer messages incorporating the extraction and the transformation of the values associated with the correlated points. The prototype system has been applied to multi-scale simulations in nano-science, in which
\end{abstract}


RISM (Reference Site Model) and FMO (Fragment Molecular Orbital) are coupled to analyze an entire electric structure of large-scale molecules immersed in infinite solvent. RISM is employed to analyze the pair correlation functions of molecular sites between a solvent and a solute, while FMO is used to calculate the total electronic energy and the molecular structure of the solute. The interoperability between nano-science applications on the grid might require the functionality to reuse application codes and data from one application domain to another as well as to retrieve and transport data. In the execution step of nano-applications, we have to focus on providing the grid-ready environment which could easily enable the execution, linkage, and coordination of the application modules and the data. To achieve this end, Grid Application Environment Team(WP3) in NAREGI has been developing Workflow Tool, GridPSE and Grid Visualization Tools in cooperation with the resource management mechanism. We expect that these middlewares and tools could be a key component for enabling gateway developers to manage and provide applications on the computational resources for execution and for analyzing linked data sets from related domains, such as Monte Carlo calculations, molecular dynamics, electronic structure studies, and further cross-disciplinary data mining. 


\section{Q\&A - Mutsumi Aoyagi}

\section{Questioner: Brian Ford}

Congratulations on your computation of a very large molecule in this way! Have you found that the computing method you have chosen confirms and supports your chemical intuitions? As the molecular groups within the ensemble rotate, do you anticipate changing the strategy of your computation?

\section{Mutsumi Aoyagi}

Thank you very much. I found that the fragment Molecular Orbital (FMO) method works very well for the decomposition on simple chemical bonds like a single bond, but for molecules having multiple-bonds or delocalized electrons, we must carefully apply the FMO method to such systems. Yes, we realize that different decomposition schemes have to be applied according to large changes of molecular structure, such as a rotation of molecular groups.

\section{Questioner: Mladen Vouk}

In your multi-scale andlor multi-physics simulation example, the large molecule is immersed in water. What happens to the possible hydrogen bonding between the molecule and "thin" water layer around it? Is it computed by the FMO code or by the water "integral" code?

\section{Mutsumi Aoyagi}

Yes, hydrogen bonding of surrounding water molecules with solute, such as large protein molecule, plays an important role in solvation dynamics of protein. So the surrounding water molecules located at the 1st layer are treated as the part of solute. In other word, hydrated protein with thin water is calculated by FMO, and surrounding water molecules located at 2nd layer are treated through statistical method in our multi-scale and/or multi-physics simulation.

\section{Questioner: Mladen Vouk}

In your slides which show decomposition of the FMO code into modules, does your grid system automatically adapt the code (through automatic recompilation) if mapping onto computing resources offers a platform for which you may not have precompiled/optimized executables?

\section{Mutsumi Aoyagi}

Our grid system, NAREGI-PSE, does not have automatic recompilation functionalities. Instead, since the compilation conditions required for each module are registered on the application information service, NAREGI-PSE can deploy our modules onto the appropriate computing resources automatically. 


\section{Questioner: Bill Applebe}

Is the water molecule display purely for one time-step?

\section{Mutsumi Aoyagi}

Yes, the calculated results correspond only to one time-step, and in order to investigate the dynamical aspect of solvated molecule, including the analysis of free energy surface, we need to iteratively solve the RISM equation for solvent according to the structural changes of solute. 